\title{
Massive cavitary pulmonary rheumatoid nodules in a patient with HIV
}

\author{
N. Khazeni*, R.J. Homer" ${ }^{\#}$ A.N. Rubinowitz" and G.L. Chupp*
}

\begin{abstract}
The case of a 52-yr-old female with rheumatoid arthritis and HIV who developed massive, progressive, cavitary pulmonary nodules is described.

Multiple diagnostic bronchoscopies and lung biopsies failed to demonstrate the presence of any microorganisms. Pathological analysis showed palisading histiocytes with necrobiosis consistent with rheumatoid nodules.

The effect of co-existing HIV infection on the course and prognosis of rheumatoid arthritis is discussed, and it is concluded that the complex relationship between these two disease processes warrants further investigation.
\end{abstract}

\section{KEYWORDS: Cavitary pulmonary nodules, HIV, rheumatoid arthritis}

\section{CASE REPORT}

A 52-yr-old female with a history of seropositive rheumatoid arthritis (RA) presented to the Yale New Haven Hospital Emergency Department (New Haven, CT, USA) with complaints of respiratory and constitutional symptoms for several months. The patient was experiencing dyspnoea on exertion, cough productive of greenish-yellow and occasionally blood-tinged sputum, anorexia, chills, subjective fevers, night sweats, and an unintentional $4.5-\mathrm{kg}$ weight loss over the previous year. She had been diagnosed with RA 5 yrs prior to presentation. Her disease was nonerosive, manifested as minimal joint symptoms in her hands, and had been well controlled on prednisone, leflunomide, sulfasalazine and naproxen. She had a history of unprotected sexual contact, a 3-pack-yr distant smoking history, and no history of travel, pets, drug use or occupational exposures. She reported that her mother, brother and sister had lung resections for unclear reasons prior to their deaths from respiratory failure.

The patient's examination was notable for a cachectic appearance and bilateral swan neck deformities of the hands. She had no peripheral rheumatoid nodules, and the remainder of her examination, including auscultation of her chest, was unremarkable. Hand and wrist radiographs revealed periarticular osteopenia and flexion deformities of the fourth and fifth proximal interphalangeal joints, with no lytic, erosive or destructive lesions. A chest radiograph and computed tomography (CT) scan revealed a large right-sided cavitary lung lesion (figs 1 and 2). Three sputum samples were negative for acidfast bacilli (AFB), mycology, Nocardia, Actinomyces and routine bacterial culture. Serum anti-histoplasma antibody was negative. HIV ELISA and subsequent Western blot assays were positive. CD4 count was 708 cells $\cdot \mathrm{mm}^{-3}$, and HIV RNA viral load was 7,000 copies $\cdot \mathrm{mL}^{-1}$. Rheumatoid factor was elevated at 24 IU (normal $<20 \mathrm{IU})$. A purified protein derivative tuberculosis skin test was positive with $7 \mathrm{~mm}$ of induration. The patient underwent flexible fibreoptic bronchoscopy, with bronchoalveolar lavage (BAL) of the right upper lobe (RUL) and right middle lobe (RML). The stains and culture results from the BAL were negative, and pathology showed nonspecific inflammation.

No treatment was initiated and the patient presented $1 \mathrm{yr}$ later with the same symptoms and an additional 9-kg unintentional weight loss. She had been followed at a local HIV clinic with repeat CD4 counts consistently $>493$ cells $\cdot \mathrm{mm}^{-3}$. She had not been diagnosed with any opportunhighly active antiretroviral therapy. A chest radiograph and $\mathrm{CT}$ scan revealed progression of her disease (fig. 3), with an increase in the size of the right cavitary lung lesion, as well as multiple new bilateral cavitary pulmonary nodules. Repeat blood and sputum cultures again showed no growth. Serum antineutrophil cytoplasmic antibody (ANCA) immunofluorescence was istic infections, nor had she been started on

\section{AFFILIATIONS}

Depts of *Internal Medicine, Section of Pulmonary and Critical Care

Medicine,

\#Pathology, and

-Diagnostic Radiology, Yale

University School of Medicine, New Haven, CT, USA.

CORRESPONDENCE

G.L. Chupp

Pulmonary and Critical Care

Medicine

Yale University School of Medicine

300 Cedar Street

TAC-441S

PO Box 208057

New Haven

CT 06520

USA

Fax: 12037853826

E-mail: geoffrey.chupp@yale.edu

Received:

December 072005

Accepted after revision:

April 062006 


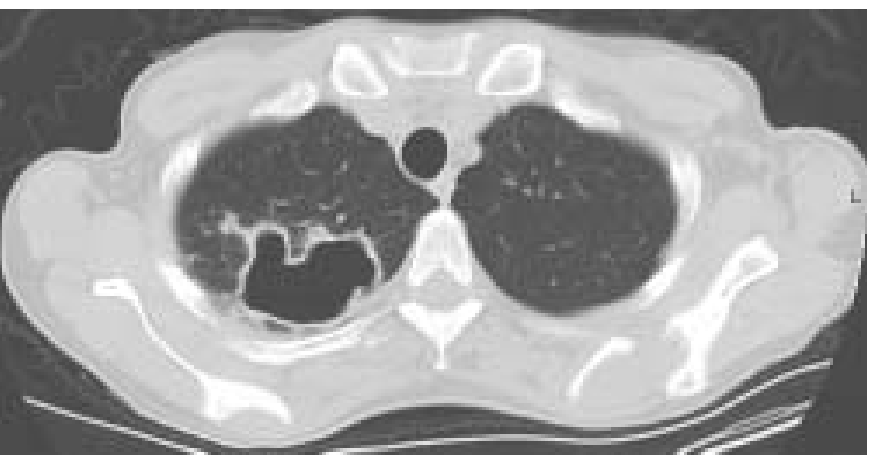

FIGURE 1. Computed tomography showing chest section through lung apices (at presentation). A few small nodules are seen adjacent to the right upper lobe cavity. The left apex appears normal.

negative. A transthoracic echocardiogram revealed no vegetations. Bronchoscopy with brushings, BAL and transbronchial biopsies (TBBx) of the RUL cavitary lesion were performed. One of the three BAL washings grew 2+ methicillin-sensitive Staphylococcus aureus (MSSA). TBBx and brushings of the RUL cavity were again negative for AFB, mycology, Nocardia, Actinomyces and routine bacterial culture. TBBx pathology revealed nonspecific inflammation. Treatment with intravenous oxacillin (Bactocill $₫$; GlaxoSmithKline, Brentford, UK) was initiated. As MSSA was not believed to be the cause of the massive cavitary lesions, the patient underwent thoracotomy with biopsy of the RML. Pathology special stains were negative for AFB and fungal forms, and histology revealed necrobiotic granulomas with palisading histiocytes (fig. 4) consistent with pulmonary rheumatoid nodules.

\section{DISCUSSION}

The differential diagnosis of cavitary pulmonary nodules includes infection with pathogens (e.g. Mycobacterium tuberculosis, Mycobacterium avium-intercellulare, Coccidiodes imitus, Histoplasmosis capsulatum, Blastomycosis dermatitidis, Cryptococcus neoformans, Actinomyces israelii, Nocardia asteroides, S. aureus, Pseudomonas aeruginosa and other virulent Gramnegative bacteria), septic pulmonary emboli, sarcoidosis,

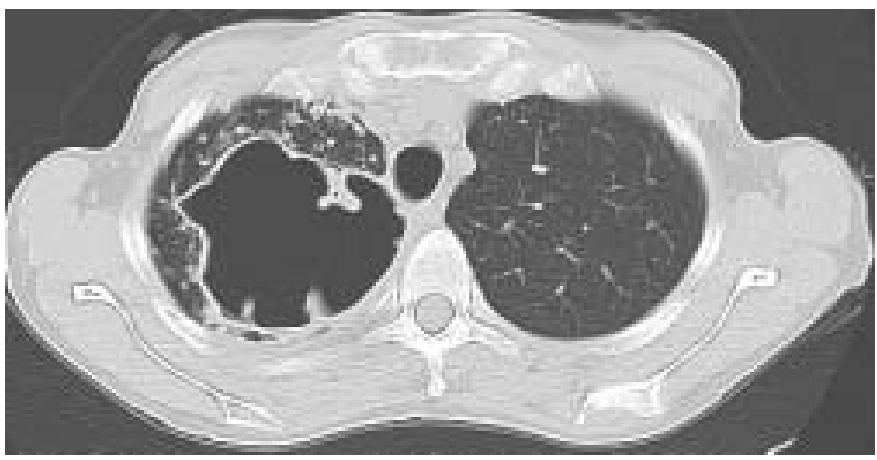

FIGURE 2. Computed tomography showing chest section through lung apices (1 $\mathrm{yr}$ after initial presentation). There is an increase in size of the right upper lobe cavity, with thick frond-like projections, and an increase in the number of nodules surrounding the cavity in the right apex.

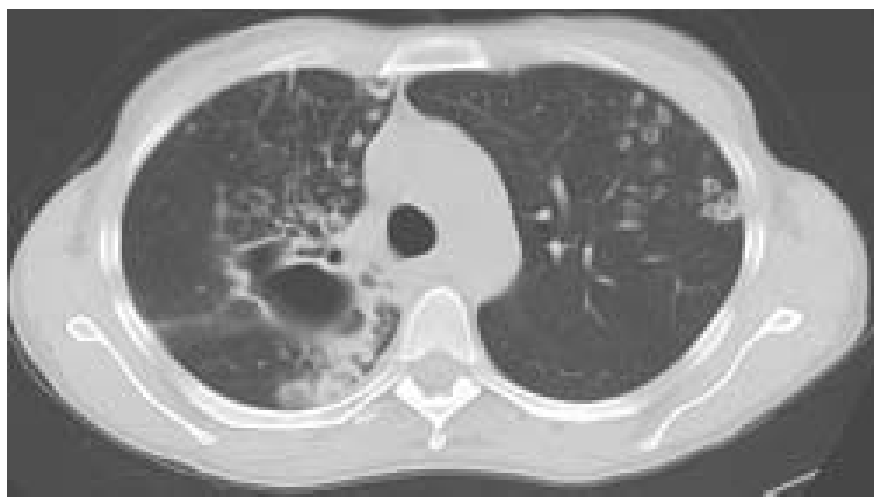

FIGURE 3. Computed tomography of the chest at the level of the aortic arch ( $1 \mathrm{yr}$ after initial presentation), showing the inferior-most extent of the right upper lobe cavity with surrounding new nodules, some of which are cavitary. There are multiple new, small, cavitary nodules in the left upper lobe.

primary lung cancers (particularly squamous cell and lymphoma), metastases (particularly squamous cell), pulmonary infarcts, Wegener's granulomatosis and pulmonary rheumatoid nodules.

The current patient's family exposure history and clinical presentation were concerning for infection, particularly with mycobacterial or fungal disease. However, her relatively slow clinical progression on immunosuppressive therapy was inconsistent with infection, and microbiological data did not support an infectious aetiology, as cultures of her RUL cavity TBBx, brushings, BAL, and nine out of 10 sputum samples failed to grow any microorganisms. Minimal growth of $M$. avium-intercellulare on one sputum culture 2 yrs after initial presentation was consistent with colonisation of abnormal pulmonary parenchyma, rather than primary infection. A normal echocardiogram and several negative blood cultures ruled out septic pulmonary emboli. Pathology was not consistent with malignancy or infarction; it did not reveal epithelioid nonnecrotising granulomas of sarcoidosis. Serum

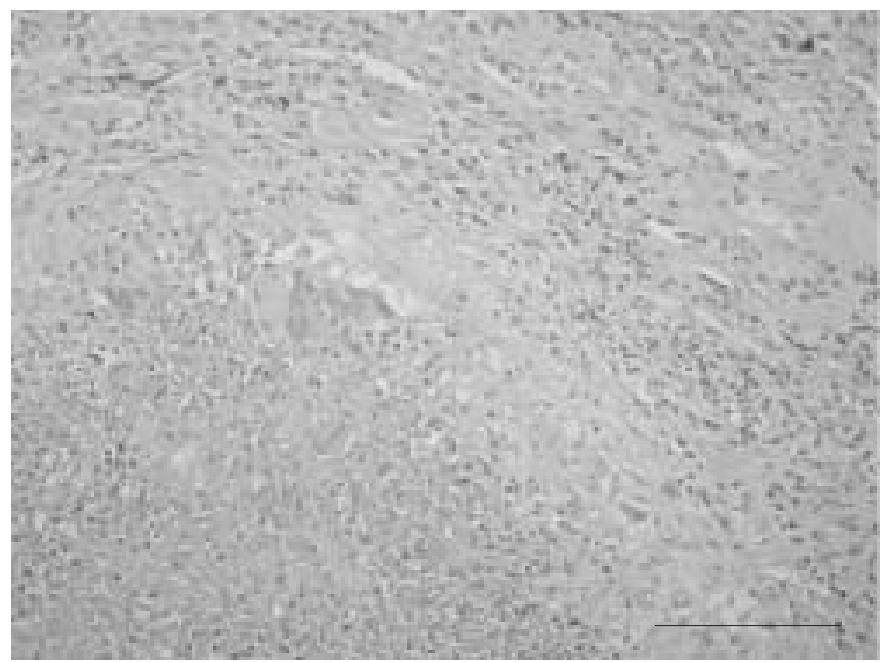

FIGURE 4. Pathology shows granulomatous inflammation with necrobiosis (lower left), palisading histiocytes (upper left to lower right) and dense scar (upper right). Haematoxylin and eosin stain. Scale bar $=100 \mu \mathrm{m}$. 
ANCAs were negative, and the patient did not develop upper respiratory or renal findings consistent with Wegener's granulomatosis. Histology showed necrobiotic pulmonary granulomas, most consistent with a diagnosis of rheumatoid nodules [1].

The current patient's presentation is consistent with that of pulmonary rheumatoid nodules in several respects: these nodules occur more commonly in patients with seropositive RA, they tend to occur as multiple bilateral nodules ranging from millimetres to several centimetres in size, they can occur and flare in the absence of systemic RA manifestations, and can cavitate $[2,3]$. The course of these nodules, however, tends to be benign [2] and, to the present authors' knowledge, there are no previously reported cases of a cavitary pulmonary rheumatoid nodule expanding to this size. It is postulated that the current patient's coexistent HIV infection may have played a role in this aggressive course of her RA-associated lung disease. Early literature cases describe patients with coexistent HIV and RA reporting complete remission of RA symptoms, and proposed the need for CD4+/CD8+ imbalances and the necessity of CD4+ T-cells for RA activity [3]; however, a growing amount of literature now suggests a more complex relationship, in which HIV may alter the typical presentation and course of RA. In 1991, the first case of active RA symptoms in a patient with HIV infection was reported [4]. A handful of cases, such as the patient presented herein, have followed. Since HIV expression can occur in CD8+ as well as CD4+ cells [5], and HIV is now known to mediate disease activity by mechanisms beyond CD4+ dysregulation, CD4+/CD8+ cell imbalances are unlikely to explain RA-HIV disease interactions in all patients. It is possible that patients infected with HIV may experience improvement in clinical symptoms of RA while immunologically driven destruction of tissues continues [6]. Furthermore, since HIV can lead to pulmonary cellular injury via induction of apoptosis [7], diseased areas of lung, such as pulmonary rheumatoid nodules, may be more susceptible to ongoing tissue injury and destruction in the setting of HIV infection.

To the current authors' knowledge, this is the first report of a rheumatoid arthritis disease manifestation dramatically worsening in a patient with coexistent HIV infection and underscores the need for further investigation into the complex relationship between these two disease processes.

\section{REFERENCES}

1 Yousem SA, Colby TV, Carrington CB. Lung biopsy in rheumatoid arthritis. Am Rev Respir Dis 1985; 131: 770-777.

2 Hunninghake G, Fauci A. Pulmonary involvement in the collagen vascular diseases. Am Rev Respir Dis 1979; 119: 471-503.

3 Bijlsma JW, Derksen RW, Huber-Bruning O, Borleffs JC. Does AIDS "cure" rheumatoid arthritis? Ann Rheum Dis 1988; 47: 350-351.

4 Kerr LD, Spiera H. The co-existence of active classic rheumatoid arthritis and HIV. I Rheumatol 1991; 18: 1739-1740.

5 Fouchard M, Reveil B, Mbayo K, et al. Evidence for HTLVIII/LAV expression by primary cultures of T8 cells. Int $J$ Cancer 1986; 38: 657-659.

6 Muller-Ladner U, Kriegsmann J, Gay RE, Koopmann WJ, Gay S, Chatham WW. Progressive joint destruction in a human immunodeficiency virus-infected patient with rheumatoid arthritis. Arthritis Rheum 1995; 38: 1328-1332.

7 Kanmogne GD, Primeaux C, Grammas P. Induction of apoptosis and endothelin-1 secretion in primary human lung endothelial cells by HIV-1 gp120 proteins. Biochem Biophys Res Commun 2005; 333: 1107-1115. 